\title{
Microdeleción 16p12.2: reporte de un caso clínico y revisión de la literatura
}

\author{
16 12.2 microdeletion: Report of a clinical case \\ and review of the literature
}

\author{
Maria A. Acosta-Aragón PhD', Valeria C. Restrepo-Arias $M D^{2}$, \\ Hasbleidy V. Riaño-Arévalo MD2
}

Resumen: en el presente manuscrito se describe el caso clínico de una adolescente con retardo mental y otras manifestaciones clínicas que en conjunto hacen sospechar de una enfermedad de origen genético, la microdeleción 16p12.2. El texto enfatiza en el abordaje diagnóstico adecuado de estos pacientes a través del análisis completo del caso y una revisión de la literatura sobre el tema.

Palabras clave: deleción cromosómica, microdeleción 16p12.2, cromosomas humanos par 16, hibridación genómica comparativa.

Abstract: In this manuscript it is described the clinical case of an adolescent with mental retardation and other clinical manifestations that together make suspect a disease of genetic origin, 16p12.2 microdeletion. The text emphasizes on proper diagnostic approach of these patients, through the comprehensive analysis of the case and a review of the literature about this issue.

Key words: Chromosome deletion, 16p12.2 microdeletion, human chromosomes pair 16, comparative genomic hybridization.

Acosta-Aragón MA, Restrepo-Arias VC, Riaño-Arévalo HV. Microdeleción 16P12.2: reporte de un caso clínico y revisión de la literatura. Medicina \& Laboratorio 2016; 22: 381-388.

\footnotetext{
${ }^{1}$ Médica, MSc en Biología, PhD en Genética de Poblaciones Humanas y Genética Forense. Integrante, Grupo de Investigación Lactancia Materna y Alimentación Complementaria, Docente Departamento de Pediatría, Facultad de Ciencias de la Salud, Universidad del Cauca. Popayán, Colombia. Correo electrónico: morin1924@gmail.com

2 Médicas y cirujanas, Universidad del Cauca. Popayán, Colombia.

Conflicto de intereses: los autores declaran que no tienen conflicto de intereses Medicina \& Laboratorio 2016; 22: 381-388

Módulo 21 (Casos clínicos), número 17. Editora Médica Colombiana S.A. $2016^{\circ}$

Recibido el 03 de junio de 2016; aceptado el 29 de agosto de 2016
} 
$\mathrm{D}$ entro de los antes denominados síndromes de genes contiguos se encuentran las microduplicaciones y microdeleciones, que es como se conocen hoy en día estos trastornos. Se cree que por cada microdeleción hay una microduplicación recíproca; no obstante, en la actualidad solo se han reportado 79 síndromes de microduplicación en comparación con los 211 síndromes de microdeleción descritos. A este último grupo pertenece la microdeleción 16p12.2 [1].

La frecuencia estimada de la microdeleción 16p12.2 en los individuos sometidos a pruebas de microarreglos es del 0,19\% y la incidencia aproximada es de 1 por cada 15.000 nacidos vivos; sin embargo, esta cifra no tiene en cuenta individuos sanos o levemente afectados heterocigotos, por lo que algunos estudios de control muestran una frecuencia mayor, de 1 por cada 1.400 a 2.000 individuos [2].

A la fecha no es claro el número de casos reportados en la literatura mundial sobre esta entidad, pero el estudio de Girirajan y colaboradores [3], realizado en 2010, proporciona una estimación de esta cifra. En este metanálisis genómico se identificó una microdeleción recurrente en el cromosoma $16 \mathrm{p} 12.2$ en 42 de 21.127 individuos con retraso en el desarrollo y discapacidad intelectual y en 8 de 14.839 controles, arrojando una asociación altamente significativa de esta microdeleción en los individuos afectados.

Por tratarse de una entidad infrecuente es poco lo que se ha descrito sobre la microdeleción del cromosoma 16 en la banda 12, pero se sabe que es un síndrome de reconocimiento limitado por su heterogeneidad clínica [2]. El presente texto tiene por objetivo describir detalladamente un caso clínico de una adolescente con este síndrome inusual, haciendo énfasis en el diagnóstico clínico y molecular, con una revisión de la literatura sobre el tema.

\section{Reporte del caso}

\section{Información del paciente}

Paciente de 13 años de edad, nacida en el municipio de Bolívar, Cauca (Colombia), quien asiste en el año 2012 al servicio de Pediatría del Hospital Universitario San José de Popayán por dificultad en el aprendizaje, trastorno en el lenguaje y «marcha agazapada». En la consulta se encuentra retraso del desarrollo, talla baja y algunos hallazgos dismorfológicos por lo que interconsulta con el servicio de Genética Clínica.

La información sobre los antecedentes familiares y la infancia se desconoce debido a que no se tiene contacto con su familia biológica y actualmente se encuentra a cargo de cuidadores sustitutos. Dentro de los antecedentes personales se destacan retardo mental, retraso global en el desarrollo psicomotor, epilepsia, diplejía 
espástica, infección TORCH (toxoplasmosis, rubéola, citomegalovirus, herpes simplex y VIH), desnutrición crónica severa y desacondicionamiento físico.

\section{Hallazgos clínicos}

En la evaluación clínica se encontró una adolescente femenina en buenas condiciones generales, con signos vitales normales, talla: $135 \mathrm{~cm}$, peso: $26 \mathrm{~kg}$, brazada: $136,5 \mathrm{~cm}$, distancia intercantal interna: $27 \mathrm{~mm}$, distancia intercantal externa: $77 \mathrm{~mm}$, filtrum: $11 \mathrm{~mm}$, oreja derecha: $5,6 \times 3 \mathrm{~cm}$, oreja izquierda: $5,8 \times 3,5 \mathrm{~cm}$, distancia intermamilar: 15 $\mathrm{cm}$ y relación segmentos superior/inferior (SS/SI): 0,8. Destacaron, además, microcefalia y desproporción del macizo craneofacial, frente estrecha, depresiones frontales laterales, hendiduras palpebrales hacia arriba, puente nasal

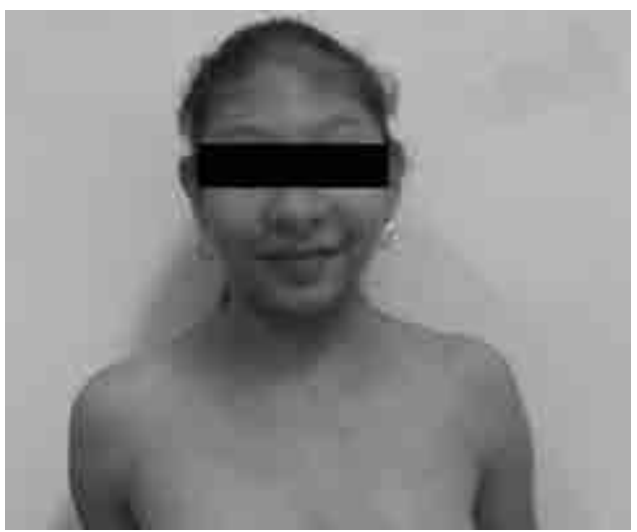

Figura 1. Paciente con algunos de los dismorfismos craneofaciales descritos, en quien resalta la microcefalia. Fotografía tomada con el consentimiento de la acudiente. ancho, tabique nasal alto e hipoplásico, cuello corto, escoliosis derecha, vello axilar escaso, desarrollo puberal Tanner IV, genu valgo, contractura bilateral en flexión de las rodillas, dedos largos y delgados e hirsutismo (véase figura $\mathbf{1}$ ).

\section{Evaluación diagnóstica}

Durante un periodo de cuatro años se llevaron a cabo los siguientes estudios:

- 2012: se realizó electroencefalograma, donde se describió actividad de puntas lentas témporo-occipitales izquierdas epileptógenas, y tomografía axial computarizada cerebral que evidencia hidrocefalia asimétrica de predominio supratentorial derecho, con disminución de los diámetros craneanos y aspecto de microcráneo (véase figura 2 ).

- 2013: se solicitó la determinación de los niveles de TSH y T4 libre para

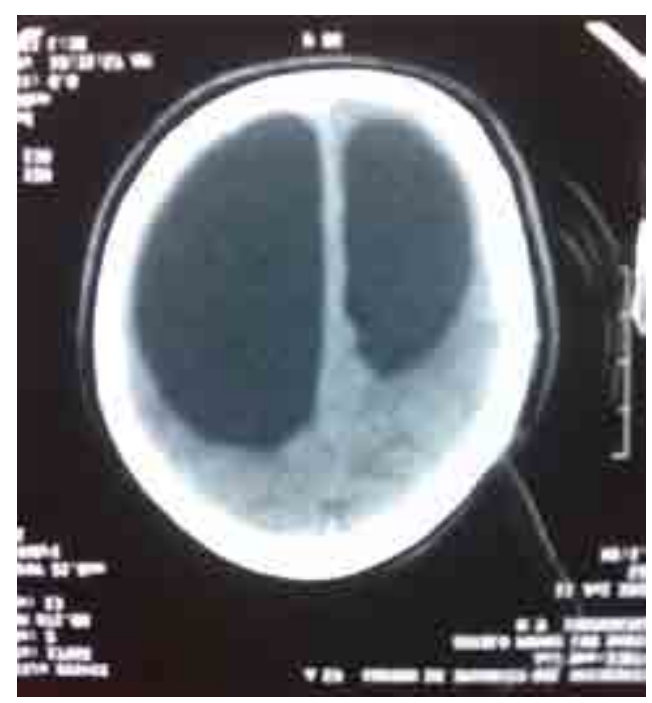

Figura 2. Tomografía axial computarizada cerebral de la paciente del caso en la que se muestra hidrocefalia asimétrica de predominio supratentorial derecho, disminución de los diámetros craneanos y aspecto de microcráneo. 
descartar endocrinopatía, los cuales fueron normales. Además, se realizó cariotipo que reportó $46 \mathrm{XX}$.

- 2014: se solicitó perfil TORCH, siendo positivo para toxoplasmosis, citomegalovirus y rubeóla; además, se tomaron niveles de la hormona del crecimiento que resultó dentro de los rangos normales. Por ortopedia se realizaron radiografías dorsolumbar, de caderas y de rodillas, que evidenciaron escoliosis dorsolumbar de vértice derecho, coxa valga y genu valgo. Este mismo año se realizó audiometría que mostró niveles sensoriales auditivos normales.

- 2015: se realizaron ecografía renal y de vías urinarias, electrocardiograma y ecocardiograma que resultaron normales. A finales de este año, ordenada por el servicio de Genética, se realizó la prueba molecular en la que se evidenció la microdeleción 16p12.2.

En cuanto al razonamiento diagnóstico se debe tener en cuenta que, a pesar de que se conocen ciertas características clínicas asociadas a este síndrome, no hay criterios clínicos establecidos y el diagnóstico diferencial es amplio, por lo que en esta paciente el diagnóstico clínico fue difícil y se requirieron pruebas moleculares para confirmar la sospecha de esta entidad.

En relación al pronóstico y partiendo de que se trata de una enfermedad cuya causa no es modificable y para la cual no existe tratamiento, el pronóstico depende del mantenimiento de la calidad de vida y la prevención de las complicaciones.

\section{Intervención terapéutica}

En las intervenciones terapéuticas y de rehabilitación han tenido participación varias especialidades como neuropediatría, nutrición clínica, fisiatría, ortopedia y traumatología y genética clínica. Desde el 2012 la paciente recibe, además, terapias integrales en neurodesarrollo, físicas y fonoaudiológicas tres veces por semana.

\section{Seguimiento y resultados}

Teniendo en cuenta los posibles resultados clínicos y sociales en ausencia de las intervenciones realizadas se podría decir que el resultado al momento es positivo tanto para la paciente como para sus cuidadores.

\section{Puntos fuertes y limitaciones en el manejo de este caso}

Una de las fortalezas en el estudio de este caso fue que la sospecha clínica y el diagnóstico diferencial adecuados permitieron el enfoque correcto del mismo. Dentro de las limitaciones la principal fue la ausencia de información sobre los antecedentes de la paciente. 


\section{Discusión}

El retardo mental aislado o asociado a malformaciones genéticas afecta entre un $2 \%$ y $3 \%$ de la población general; sin embargo, muchas de las causas que lo originan aún se desconocen, de modo que sólo se identifica su origen en la mitad de los pacientes afectados. En cuanto al retardo mental grave se alcanza el diagnóstico en una proporción cercana al 70\% [4,5]. Muchos síndromes asociados al retardo mental son causados por deleciones y raramente por duplicaciones. Los pacientes con deleciones pueden presentar, además, retraso del desarrollo, un fenotipo conductual característico y múltiples anomalías físicas $[1,5]$.

De esta manera inició la sospecha clínica en la paciente presentada en este reporte de caso; una adolescente con retardo mental, retraso en el desarrollo y ciertos hallazgos dismorfológicos, con cariotipo 46XX. En este aspecto se debe recordar que en muchos casos, como este, el cariotipo puede ser normal, por lo que se han venido desarrollando nuevas técnicas que permiten detectar reorganizaciones que no son alcanzadas por la resolución del microscopio óptico $[5,6]$. Entre estas reorganizaciones se encuentra la microdeleción 16p12.2, que consiste en la pérdida de un segmento cromosómico que alberga siete genes y está asociada a duplicaciones segmentarias, con grandes bloques de secuencias repetidas, con más del $90 \%$ de identidad de secuencia y que contienen seis genes adicionales $[2,7,8]$.

La localización de esta microdeleción, originalmente descrita en 16p12.1 (de coordenadas 21.850.000-22.370.000), ha cambiado a 16p12.2 (de coordenadas 21.950.000-22.470.000), por lo que los informes clínicos ahora utilizan esta última ubicación [2,9]. Esta microdeleción se expande aproximadamente $500 \mathrm{kB}$, lo que lleva a un estado hemicigoto de seis genes: UQCRC, C15orf65, VWA3A, eEF2K, POLR3E y CDR2 [7,8] (véase figura 3). En la paciente presentada en este caso se detectó la deleción 16p12.2 con coordenadas CHC16: 21.950.36022.420.365 y que abarcaba los siguientes genes: UQCRC2, PDZD9, VWA3A, EEF2K, C160M52, POLR3E y COR, lo que coincide con lo descrito previamente en la literatura para esta entidad.

En cuanto a las manifestaciones clínicas muchas se han asociado con esta supresión y, aunque no todas son totalmente reconocibles, casi siempre se relacionan con retraso en el desarrollo y problemas de aprendizaje $[1,7,10]$, entre los que se incluyen discapacidad intelectual, retraso del desarrollo, el crecimiento y el habla, trastornos del sueño, hipotonía, anomalías craneofaciales, microcefalia y convulsiones $[2,7,8]$. Es de recordar que la mayoría de estas características estuvieron presentes en la paciente de este reporte de caso, en la que, por otro lado, se descartaron alteraciones psiquiátricas y malformaciones cardiacas que también se describen en la literatura para esta entidad $[2,11]$. 


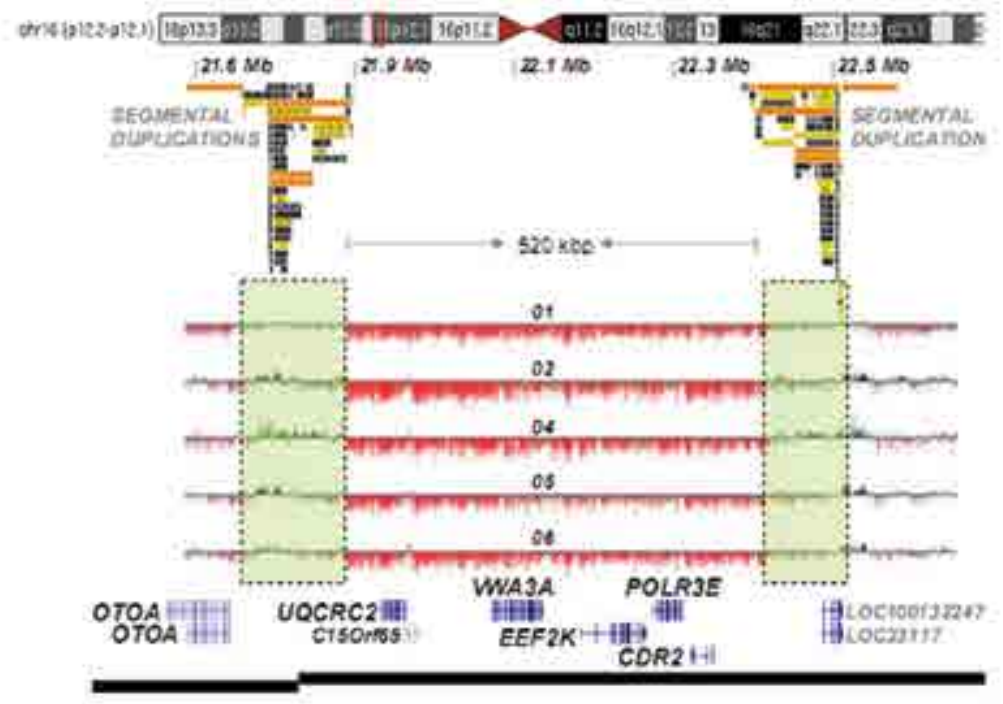

Figura 3. Microdeleción 16p12.2. En la imagen se muestra la expansión de aproximadamente $500 \mathrm{kB}$ y el estado homocigoto de seis genes: UQCRC, C15orf65, VWA3A, eEF2K, POLR3E y CDR2. Tomado de: 16 12.2 deletion study, en Girirajan Lab, n.d. Consultado enero 16, 2016, en https://autism.bx.psu. edu/16p12.2/about.html. Copyright ${ }^{\odot} 2016$ por The Girirajan Lab.

Para la microdeleción $16 \mathrm{p} 12.2$ no hay establecidos criterios diagnósticos formales y, debido a la presentación variable, se realiza, como se mencionó antes, mediante análisis a nivel molecular con estudio de microarreglos u otros análisis genómi$\cos [2,5,7]$. Referente al tratamiento se debe dirigir a los problemas específicos identificados, por lo que se recomienda la derivación a otros especialistas con base en la clínica del paciente [2].

En cuanto al modo de herencia la microdeleción $16 \mathrm{p} 12.2$ se hereda de forma autosómica dominante. La mayoría (95\%) de personas que la poseen la heredan de un padre que puede o no tener las características clínicas $[2,3,12]$; sólo se ha descrito un caso con una microdeleción de novo, pero sin pruebas de paternidad confirmatorias [2]. En el caso actual no fue posible la realización de estudios en familiares para definir el tipo de herencia.

Se ha descrito que los padres con la microdeleción son propensos a tener convulsiones, retraso mental leve y problemas psiquiátricos, lo que sugiere que este genotipo es un factor de riesgo para el desarrollo de fenotipos con penetrancia incompleta y expresividad variable. Cada hijo de una persona con la deleción tiene una probabilidad del $50 \%$ de heredarla y el riesgo para la descendencia afectada es menor del $50 \%$ debido a la penetrancia incompleta $[2,3,12]$.

Por último, en cuanto al diagnóstico diferencial se puede decir que es amplio e incluye todas las causas de retraso en el desarrollo $[1,4,10]$. Los principales diagnósticos diferenciales a tener en cuenta son los síndromes relacionados con el 
cromosoma 16, entre ellos se encuentran dos de especial interés: a) el síndrome de deleción 16p12.2-p11.2, que se caracteriza por retraso en el desarrollo y deterioro cognitivo, facies plana, fisuras palpebrales oblicuas hacia abajo, orejas de implantación baja, hendidura orofacial y defectos cardíacos $(1,10)$ y b) el síndrome de duplicaciones recíprocas 16p12.2-p11.2, que se ha reportado en pacientes con autismo y rasgos dismórficos como cara redonda, macrostomía y macrocefalia $[1,4]$.

Existen otras entidades para tener en cuenta en el diagnóstico diferencial como el retardo mental de origen cromosómico, el cual se asocia con retardo mental grave y fenotipo polimalformativo [4], el síndrome de Smith Magenis o deleción 17p11.2, que se caracteriza por retardo mental, braquicefalia, hipoplasia mediofacial, mandíbula prominente, voz ronca, alteraciones del sueño y compulsión por la onicofagia $[10,13]$ y, por último, el síndrome de Miller Dieker o microdeleción 17p13.3, en el que se agrupan características como retardo mental severo, convulsiones, lisencefalia, diámetro bifrontal estrecho, frente alta, nariz pequeña, narinas antevertidas, labio superior fino y cardiopatía [4,14]. Para finalizar se deben tener en cuenta las entidades relacionadas con microcefalia, entre ellas el síndrome de Angelman, en el que las personas afectadas presentan retardo mental severo, ausencia de lenguaje, movimientos atáxicos, conducta especial y convulsiones, con un sistema nervioso estructuralmente normal $[4,15,16]$.

Todas las anteriores entidades se asocian al retardo mental y el retraso en el desarrollo y muchas de las características presentes en ellas se superponen con las del caso analizado, lo que pone en evidencia la dificultad en el diagnóstico basado sólo en la clínica y la necesidad de realizar pruebas específicas que permitan confirmar el diagnóstico. Así se llevó a cabo el enfoque de esta paciente, empezando por la historia genética y la evaluación clínica adecuada, pasando por la elaboración de un buen diagnóstico diferencial hasta lograr la realización de una prueba molecular que confirmara la sospecha.

\section{Conclusiones}

Teniendo en cuenta los aspectos descritos en la literatura el presente caso clínico hace referencia a un paciente $46 \mathrm{XX}$ con síndrome de microdeleción 16p12.2, confirmado con hibridación genómica comparada (microarreglos), con sospecha diagnóstica inicial debido a la presentación de varias manifestaciones clínicas como retardo mental, retraso en el desarrollo y talla baja; además de otros dismorfismos como anomalías craneofaciales y esqueléticas, sin que se documentara en la paciente alteraciones visuales, auditivas, cardiacas, renales ni alteraciones psiquiátricas o de comportamiento. Actualmente, la paciente se encuentra en seguimiento y manejo multi e interdisciplinario por especialidades que pretenden mejorar y mantener la calidad de vida del caso índice y su familia adoptiva. 


\section{Bibliografía}

1. Weise A, Mrasek $K$, Klein E, Mulatinho M, Llerena JC, Jr., Hardekopf D, et al. Microdeletion and microduplication syndromes. J Histochem Cytochem 2012; 60: 346-358.

2. Girirajan S, Moeschler J, Rosenfeld J. 16p12.2 Microdeletion. In: Pagon RA, Adam $\mathrm{MP}$, Ardinger $\mathrm{HH}$, Wallace SE, Amemiya A, Bean LJ, et al. eds. GeneReviews $\AA$ [Internet]. Washington, Estados Unidos: University of Washington; 2015.

3. Girirajan S, Rosenfeld JA, Cooper GM, Antonacci $F$, Siswara $P$, Itsara $A$, et al. A recurrent $16 \mathrm{p} 12.1$ microdeletion supports a two-hit model for severe developmental delay. Nat Genet 2010; 42: 203-209.

4. Guitart-Feliubadalo $\mathbf{M}$, Brunet-Vega $\mathbf{A}$ Villatoro-Gomez S, Baena-Diez N, Gabau-Vila E. [Chromosomal causes that produce mental retardation: chromosome disorders that can be diagnosed in the patient]. Rev Neurol 2006; 42 Suppl 1: S21-26.

5. Constanso $\mathbf{C}$, Rodríguez $\mathbf{P}$, Mosquera R Actualización de un estudio coste-efectividad de arrays de hibridación genómica comparada para el diagnóstico de retraso mental y/o del desarrollo. Gest y Eval Cost Sanit 2013; 14 51-69.

6. Antonacci $\mathbf{F}$, Kidd $\mathbf{J M}$, Marques-Bonet $\mathbf{T}$, Teague B, Ventura M, Girirajan S, et al. A large and complex structural polymorphism at $16 \mathrm{p} 12.1$ underlies microdeletion disease risk. Nat Genet 2010; 42: 745-750.

7. Santhosh Girirajan Lab. The 16p12.2 Deletion Project. 2014. Disponible: http://bx.psu. edu/girirajan lab/16p12.2/about deletion. html. Consultado: agosto 2016.

8. Online Mendelian Inheritance in Man (OMIM), Johns Hopkins University. Chromosome 16p12.1 Deletion Syndrome, 520-
KB. 2016. Disponible: http://www.omim.org/ entry/136570. Consultado: agosto de 2016.

9. Online Mendelian Inheritance in Man (OMIM), Johns Hopkins University. Chromosome 16p12.2-p11.2 Deletion Syndrome, 7.1- TO 8.7-MB. 2016. Disponible: http:// www.omim.org/entry/613604. Consultado: agosto de 2016 .

10. Vogels A, Fryns J-P. Microdeleciones y genética molecular. Atlas of Genetics and Cytogenetics in Oncology and Haematology. 2004. Disponible: http://atlasgeneticsoncology.org/ Educ/MicrodelecionesSpID30059SS.html. Consultado: ene 2016.

11. D'Alessandro LC, Werner $\mathbf{P}, \mathbf{X i e}$ HM, Hakonarson $\mathbf{H}$, White PS, Goldmuntz $\mathbf{E}$. The prevalence of $16 \mathrm{p} 12.1$ microdeletion in patients with left-sided cardiac lesions. Congenit Heart Dis 2014; 9: 83-86.

12. Veltman JA, Brunner HG. Understanding variable expressivity in microdeletion syndromes. Nat Genet 2010; 42: 192-193.

13. Lacombe D, Moncla A, Malzac P, Mattei MG, Battin J. [Smith-Magenis syndrome]. Arch Pediatr 1997; 4: 438-442.

14. Matarese CA, Renaud DL. Classical (type I) lissencephaly and Miller-Dieker syndrome. Pediatr Neurol 2009; 40: 324-325.

15. Kalsner L, Chamberlain SJ. Prader-Willi, Angelman, and 15q11-q13 Duplication Syndromes. Pediatr Clin North Am 2015; 62: 587-606.

16. Valente KD, Varela MC, Koiffmann $\mathbf{C P}$, Andrade JQ, Grossmann R, Kok F, et al. Angelman syndrome caused by deletion: a genotype-phenotype correlation determined by breakpoint. Epilepsy Res 2013; 105: 234239. 